\title{
Standards und Richtlinien für Hospiz- und Palliativversorgung in Europa: Teil $\mathbf{2}^{\mathbf{1}}$ Weißbuch zu Empfehlungen der Europäischen Gesellschaft für Palliative Care (EAPC)
}

\author{
White Paper on Standards and Norms for Hospice and Palliative Care in Europe: Part 2 \\ Recommendations of the European Association for Palliative Care
}

\author{
Autoren \\ L. Radbruch ${ }^{1}$, S. Payne ${ }^{2}$ \\ Übersetzt von D. Büche, E. Schmidlin, S. Jünger
}

Institute

Klinik für Palliativmedizin, Universitätsklinikum Bonn und Zentrum Palliativmedizin, Malteser Krankenhaus Bonn/Rhein-Sieg
European Association for Palliative Care (EAPC) President
und der EAPC-Vorstand (Michaela Bercovitch, Augusto Caraceni, Tine De Vlieger, Pam Firth, Katalin Hegedus, Maria Nabal,
André Rhebergen, Esther Schmidlin, Per Sjøgren, Carol Tishelman, Chantal Wood, and Honorary Director Franco De Conno)

Schlüsselwörter

- Standards

- Normen

- Qualität

- Delphi Prozedur

- European Association

for Palliative Care

\section{Keywords}

- standards

- norms

- quality

- Delphi procedure

- European Association for Palliative Care

\section{Bibliografie}

DOI http://dx.doi.org/

10.1055/s-0031-1276957

Z Palliativmed 2011; 12:

260-270

(c) Georg Thieme Verlag KG

Stuttgart · New York .

ISSN 1615-2921

Korrespondenzadresse Univ.-Prof. Dr. Lukas Radbruch Klinik für Palliativmedizin, Universitätsklinikum Bonn Tel.: +49-228-287-13495 Zentrum Palliativmedizin, Malteser Krankenhaus Bonn/ Rhein-Sieg

Von-Hompesch-Straße 1

53123 Bonn

Tel.: +49-228-64819170

Fax: +49-228-64819209

lukas.radbruch@malteser.org

\section{Zusammenfassung}

$\nabla$

Die Europäische Gesellschaft für Palliative Care (EAPC) hat den Schwerpunkt dieser Richtlinie auf standardisierte Normen für Hospize und Palliative Care in Europa gelegt. Der Inhalt richtet sich an die Gesellschaften und das Personal, die direkt mit der Palliativversorgung zu tun haben. Der zweite Teil dieses offiziellen Positionspapiers der EAPC stellt die generellen Anforderungen an Angebote der Palliativversorgung sowie die spezifischen Anforderungen an die verschiedenen Arten von Versorgungsangeboten dar. Themenrelevante Inhalte werden in einer Reihe zukünftiger Publikationen detaillierter behandelt.

... Fortsetzung aus European Journal of Palliative Care (EJPC) 2009; 16.6

\section{Abstract \\ $\nabla$}

The focus of this White Paper is on norms and standards for hospice and palliative care in Europe as recommended by the EAPC. The content has been restricted to those issues directly related to the distribution of services in the community as well as the material, staffing and personal equipment of these services. Part two of this official position paper of the EAPC outlines general requirements for palliative care services and the specific requirements for each service type. Relevant topics will be examined in more depth in a series of future papers.

... Continued from the European Journal of Palliative Care (EJPC) 2009; 16.6

\section{Umsetzung der Palliativversorgung}

In diesem Abschnitt steht die Umsetzung von Palliativversorgung in Wohnortnähe im Mittelpunkt. Die Basisvoraussetzungen und grundlegende Anforderungen an palliative Versorgungsangebote werden beschrieben. Anschließend werden in Teil 8 die verschiedenen Arten von Versorgungsangeboten mit den jeweils spezifischen Anforderungen an sie vorgestellt.

Die folgenden grundlegenden Anforderungen sind für die Bereitstellung von Palliativversorgung in hohem Maße relevant:

- vorausschauende Planung

\footnotetext{
${ }^{1}$ Translated from the article „White Paper on standards and norms for hospice and palliative care in Europe: part 2“ first published in the European Journal of Palliative Care 2010; 17 (1): 22-33, continued from the European Journal of Palliative Care 2009; 16 (6): 278-289. Kindly reproduced by permission of the publishers of the EJPC, who retain the copyright. All rights reserved. www.ejpc.eu.com

Übersetzung des Artikels „White Paper on standards and norms for hospice and palliative care in Europe: part 2“.
}

- Kontinuität der Versorgung

- Verfügbarkeit der Versorgung

bevorzugter Ort der Versorgung

Im folgenden Text heben die fettgedruckten Textpassagen die Empfehlungen der EAPC hervor.

\subsection{Vorausschauende Planung \\ (Advance Care Planning)}

Idealerweise besprechen sich der Patient ${ }^{2}$, die Familie und das Palliativteam bezüglich der Planung und Umsetzung der Palliativversorgung miteinander und berücksichtigen dabei die Präferenzen und Ressourcen des Patienten sowie die medizinischen Empfehlungen. Änderungen des Zu-

Erstpublikation im European Journal of Palliative Care 2010; 17 (1): 22-33. Fortsetzung von „Teil 1“, Erstpublikation im European Journal of Palliative Care 2009; 16 (6): 278-289, deutsche Übersetzung in Z Palliativmed 2011; 12: 216-227. Mit freundlicher Genehmigung der Herausgeber der EJPC, die das Copyright behalten. Alle Rechte vorbehalten. www.ejpc.eu.com

2 Zur Verbesserung der Lesbarkeit erfolgen alle Personenbezeichnungen wie „Patient" und „Mitarbeiter“ nur in männlicher Form. 
stands oder der Leistungsfähigkeit des Patienten werden durch entsprechende Änderungen der Behandlung berücksichtigt; aus einem Prozess kontinuierlicher Diskussion und Anpassung ergibt sich der individuelle, den Krankheitsstadien entsprechende, Behandlungsplan.

Mit dem Fortschreiten der Erkrankung und einer Verschlechterung der kognitiven und körperlichen Funktionen kann die Entscheidungsfähigkeit mancher Patienten jedoch eingeschränkt sein, sodass sie möglicherweise nicht mehr fähig sind, an der Entscheidungsfindung mitzuwirken. Daher sollte die Palliativversorgung die Methoden der vorausschauenden Planung einbeziehen, damit diese Patienten eine ihren Vorstellungen entsprechende Palliativversorgung erhalten können, falls sie es wünschen.

Patientenverfügungen erlauben es den Patienten, ihre persönliche Autonomie zu bewahren und geben Anleitung zur Versorgung, falls die Patienten urteilsunfähig werden und keine Entscheidungen mehr treffen können [1].

Der rechtliche Stellenwert der Patientenverfügungen ist in den europäischen Ländern unterschiedlich. Nur in wenigen Ländern sind diese Dokumente nach geltendem Recht verbindlich.

Es wird fortlaufend diskutiert, wie verbindlich Patientenverfügungen sein sollten. Die Präferenzen eines Patienten könnten sich mit dem Fortschreiten der Erkrankung verändern, und es ist auch nicht immer klar, ob die Patienten angemessen informiert wurden oder die entsprechenden Informationen verstanden haben. Somit könnten Patientenverfügungen für eine zu einem bestimmten Zeitpunkt bestehende Situation nicht mehr maßgeblich oder nie für diese Situation gedacht gewesen sein.

Patientenverfügungen können durch eine Vorsorgevollmacht ergänzt oder ersetzt werden. Dieses Dokument erlaubt es dem Patienten, einen Stellvertreter zu benennen, eine Person, die Behandlungsentscheidungen für den Patienten treffen wird, falls der Patient unfähig wird, solche Entscheidungen zu treffen [1].

Der adäquate Einsatz sowohl eines Vorsorgebevollmächtigten als auch einer Patientenverfügung erfordert, dass der Patient und der Stellvertreter die persönlichen Präferenzen des Patienten unter Berücksichtigung der spezifischen Erkrankung besprochen haben und dass sie seitens des Palliativteams angemessen über den zu erwartenden Krankheitsverlauf informiert wurden. In einigen europäischen Ländern gibt es hierfür noch keine rechtliche Basis.

\subsection{Zugang zu Palliativdiensten}

Palliativdienste und -einrichtungen sollten für alle Patienten unverzüglich zur Verfügung stehen, wann immer und wo immer sie diese benötigen $[2,3]$.

Palliativteams sollten auf einen gleichberechtigten Zugang zur Palliativversorgung für alle Patienten hinarbeiten, unabhängig von deren Alter und Diagnosen, den jeweiligen Versorgungsstrukturen (auch in ländlichen Räumen) sowie von ethnischer Herkunft, sexueller Orientierung oder finanziellen Möglichkeiten [4].

Ein gleichberechtigter Zugang zu Palliativversorgung sollte in allen europäischen Ländern gewährleistet sein. Die Palliativversorgung sollte bedarfsorientiert und ungeachtet des kulturellen, ethnischen und sonstigen Hintergrunds gesichert sein.

Patienten mit gleichwertigen Bedürfnissen sollten in gleicher Weise zu Versorgungsangeboten Zugang haben. Der Zugang sollte nicht von der Zahlungsfähigkeit abhängen [3].

Der Zugang zu hochqualitativer Palliativversorgung sollte nicht von der Zahlungsfähigkeit des Patienten oder der Angehörigen abhängen.

\subsection{Kontinuität der Versorgung}

Die Kontinuität der Versorgung im gesamten Krankheitsverlauf und in verschiedenen Einrichtungen des Gesundheitssystems ist eine Grundvoraussetzung für Palliativversorgung [4].

Dies gilt sowohl für die Kontinuität der Versorgung während des gesamten Krankheitsverlaufes als auch hinsichtlich verschiedener Einrichtungen des Gesundheitssystems. Diese Kontinuität trägt dazu bei, Fehler in der Versorgung und unnötiges Leiden von Patienten mit einer lebensbedrohlichen Erkrankung zu vermeiden; sie kann den Patient und seine Familie davor bewahren, sich alleingelassen zu fühlen und kann sicherstellen, dass die Wünsche und Präferenzen des Patienten respektiert werden.

Palliativversorgung ist ein integraler Bestandteil aller Strukturen des Gesundheitssystems (Krankenhaus, Notfallstation, Pflegeheim, Betreuung zu Hause, betreutes Wohnen, Ambulanzen und Versorgungszentren sowie Einrichtungen außerhalb des klassischen Gesundheitssektors wie Schulen und Gefängnisse). Das Palliativteam arbeitet mit professionellen Behandlern und informellen Betreuenden in jeder dieser Versorgungsstrukturen zusammen, um die Koordination, Kommunikation und Kontinuität der Palliativversorgung über alle institutionellen und häuslichen Versorgungsstrukturen hinweg sicherzustellen. Krisen und unnötigen Verlegungen vorzubeugen, ist ein wichtiges Qualitätsmerkmal von Palliativversorgung [4].

\subsection{Bevorzugter Ort der Versorgung}

Die meisten Patienten möchten im eigenen Zuhause betreut werden, falls möglich bis zu ihrem Tod. Im Gegensatz dazu sterben die meisten Patienten in einem Krankenhaus oder einem Pflegeheim.

Eine zunehmende Datenmenge sowohl auf der Grundlage von Bevölkerungsumfragen als auch anhand von Patientenbefragungen zeigt, dass die Mehrheit der Tumorpatienten es vorziehen würde, zu Hause zu sterben. Studien haben ergeben, dass rund 75\% der Befragten sich wünschen, ihre letzte Lebensphase zu Hause zu erleben [5]. Dieser Wunsch ist jedoch an das Vorhandensein eines starken sozialen und familiären Netzwerks gebunden; der Prozentsatz jüngerer, alleinstehender oder verwitweter Patienten, die es bevorzugen würden, zu Hause zu sterben, scheint deutlich darunter zu liegen.

Im Gegensatz zu den Präferenzen der Patienten ist in Europa jedoch das Krankenhaus oder Pflegeheim am häufigsten der Sterbeort. In einer Übersichtsarbeit zur Palliativversorgung in Deutschland variierten die Angaben zum Sterbeort in Abhängigkeit von der Herangehensweise der verschiedenen Autoren zum Teil beträchtlich, das Sterben in Institutionen wurde jedoch insgesamt mit einer Häufigkeit von 60-95\% beziffert. Auf der Grundlage unterschiedlicher Studien mit variierenden Prozentsätzen kommen die Autoren der Übersichtsarbeit hinsichtlich des Sterbeorts in Deutschland zu folgenden Schätzungen: Krankenhaus 42-43\%, zu Hause 25-30\%, Alters- und Pflegeheime 15-25\%, stationäre Hospize 1-2\%, andere 3-7\% [6].

Vielen Patienten ist es möglich, die überwiegende Zeit des letzten Lebensjahres zu Hause zu verbringen, sie werden aber dann zum Sterben ins Krankenhaus verlegt. Es wurde kritisiert, dass viele sterbende Menschen aufgrund inadäquater Unterstützung im erweiterten häuslichen Umfeld zur Versorgung am Lebensende ins Krankenhaus verlegt werden [3].

Der bevorzugte Betreuungs- und Sterbeort des Patienten sollte anerkannt und mit dem Patienten und der Familie besprochen werden, und es sollten Maßnahmen getroffen werden, um diesen Präferenzen, falls möglich, gerecht zu werden [7]. 


\begin{tabular}{|c|c|c|c|c|}
\hline & \multicolumn{4}{|c|}{ Palliativversorgung } \\
\hline & $\begin{array}{c}\text { palliativer } \\
\text { Versorgungsansatz }\end{array}$ & \multicolumn{2}{|c|}{$\begin{array}{l}\text { spezialisierte Unterstützung für } \\
\text { allgemeine Palliativversorgung }\end{array}$} & $\begin{array}{c}\text { spezialisierte } \\
\text { Palliativversorgung }\end{array}$ \\
\hline akute Versorgung & Krankenhaus & \multirow{2}{*}{\multicolumn{2}{|c|}{$\begin{array}{c}\text { palliativer } \\
\text { Konsildienst }\end{array}$}} & \multirow{2}{*}{ Palliativstation } \\
\hline \multirow{2}{*}{ Langzeitversorgung } & Alten- und & & & \\
\hline & Pflegeheim & \multirow{2}{*}{$\begin{array}{l}\text { ehrenamtliche } \\
\text { Hospizdienste }\end{array}$} & \multirow[b]{2}{*}{$\begin{array}{c}\text { ambulante } \\
\text { Palliativteams }\end{array}$} & stationäres Hospiz \\
\hline Home Care & $\begin{array}{l}\text { niedergelassene } \\
\text { (Fach-)Ärzte } \\
\text { ambulante Kranken- } \\
\text { pflegedienste }\end{array}$ & & & Tageshospiz \\
\hline
\end{tabular}

Abb.1 Stufenmodell der Palliativdienste (Quelle: Nemeth C, Rottenhofer I. Abgestufte Hospiz- und Palliativversorgung in Österreich. Wien: Österreichisches Bundesinstitut für Gesundheitswesen; 2004).
Es ist jedoch wichtig, sich klarzumachen, dass der Sterbeort von anderen Faktoren als der Präferenz des Patienten bestimmt werden kann. Im Endstadium einer Erkrankung kann sich der medizinische Zustand in solchem Maße verschlechtern, dass zur intensiven medizinischen und pflegerischen Betreuung eine Krankenhauseinweisung oder Verlegung auf eine spezialisierte Station erforderlich wird, da diese Versorgung im häuslichen Umfeld nicht mehr erbracht werden kann.

\subsection{Orte der Versorgung}

Palliative Behandlung, Betreuung und Unterstützung werden im häuslichen Umfeld, in Pflegeheimen, in Altersheimen, in Krankenhäusern und in stationären Hospizen oder bei Bedarf in anderen Versorgungsstrukturen erbracht [2].

Es ist gegenwärtig anerkannt, dass Palliativversorgung auf lokaler und regionaler Ebene in Form eines „Kontinuums der Spezialisierung“ angeboten werden sollte [8]. Das Spektrum sollte sich erstrecken von Angeboten der Primärversorgung bis hin zu spezialisierten Versorgungsangeboten, deren Kernaktivität ausschließlich Palliativversorgung ist. Palliativversorgung kann angeboten werden als ein palliativer Versorgungsansatz oder als allgemeine Palliativversorgung im Rahmen nicht spezialisierter Strukturen und Einrichtungen oder als spezialisierte Palliativversorgung [2].

Nicht spezialisierte Angebote der Palliativversorgung sind:

- Gemeindekrankenpflege/spitalexterne Krankenpflege

- niedergelassene Hausärzte

- ambulante Pflegedienste

- Abteilungen des Allgemeinkrankenhauses

- Pflegeheime

Spezialisierte Angebote der Palliativversorgung sind:

- Palliativstationen

- stationäre Hospize

- palliative Konsildienste im Krankenhaus

- ambulante Palliativdienste/Home Care Teams

- ambulante Hospizdienste

- Tageshospize

- „Krankenhaus zu Hause“3

- Ambulanzen ${ }^{4}$ und Versorgungszentren

Gegenwärtig, wie auch in Zukunft, wird ein großer Teil der Palliativversorgung durch nicht spezialisierte Versorgungsangebote geleistet. Folglich müssen nicht spezialisierte Behandler einen einfachen Zugang zu fachlicher Beratung und Unterstützung durch Spezialisten haben.

\footnotetext{
${ }^{3}$ Dieses Versorgungsangebot „Hospital at home“ ist nicht in allen europäischen Ländern verfügbar.

${ }^{4}$ In der Schweiz ist anstatt Ambulanzen eher der Begriff „Ambulatorien“ gebräuchlich.
}

Nicht spezialisierte Dienstleister benötigen eine spezifische Anleitung, um den palliativen Versorgungsansatz umzusetzen oder allgemeine Palliativversorgung gewährleisten zu können. Integrative Versorgungswege sind hilfreich, um die Umsetzung zu vereinfachen [9]. Der Liverpool Care Pathway for the Dying Patient (LCP) wurde in der Leitlinie für Supportive and Palliative Care des National Institute for Health and Clinical Excellence (NI$\mathrm{CE}$ ) als Methode empfohlen, um die besonderen Bedürfnisse sterbender Tumorpatienten zu identifizieren und adäquat darauf einzugehen [7].

Integrative Versorgungswege und -modelle wie der Liverpool Care Pathway for the Dying Patient werden als Fortbildungswie auch Qualitätssicherungsinstrument zur Verbesserung der Betreuung von sterbenden Patienten in nicht auf Palliativversorgung spezialisierten Versorgungsstrukturen empfohlen.

In einigen nicht spezialisierten Versorgungsstrukturen (z.B. Radiologie, Radiotherapie oder Chirurgie) können begrenzte Ressourcen zur Einführung einer Warteliste führen. Diese Wartelisten sind für Palliativpatienten aufgrund der eingeschränkten Lebensspanne, in der sie noch von der Behandlung profitieren könnten, besonders nachteilig. Aus diesem Grund wurden „Fast Tracking Pathways“ vorgeschlagen, um Palliativpatienten bevorzugten Zugang zu gewähren [2].

In Anbetracht der verringerten Lebenserwartung der Palliativpatienten sollten in medizinischen Versorgungsstrukturen beschleunigte Behandlungspfade eingeführt werden, die eine angemessene Bevorzugung dieser Patienten sicherstellen, um einer unverhältnismäßigen Belastung durch Zeitverlust vorzubeugen.

Das Angebot an spezialisierter Palliativversorgung sollte so strukturiert sein, dass es den Patienten den klinischen Erfordernissen oder persönlichen Präferenzen entsprechend den Wechsel von einer Versorgungsstruktur in eine andere ermöglicht. Dies impliziert, dass spezialisierte palliative Versorgungsangebote keine isolierten Einheiten sind und dass die Koordination der Versorgungsangebote wesentlich ist.

Es sollte ein umfassendes System von Dienstleistungen mit stationären und ambulanten Versorgungsangeboten sowie unterstützenden Angeboten zur Verfügung stehen, um alle Versorgungsbedürfnisse und Behandlungsmöglichkeiten abzudecken [10].

In einem abgestuften System palliativer Versorgungsangebote, wie in Abb. 1 dargestellt, können die verschiedenen Bedürfnisse der Patienten und Angehörigen mit dem am besten geeigneten Versorgungsangebot in Einklang gebracht werden [10]. In einem solchen System kann der richtige Patient zum richtigen Zeitpunkt am richtigen Ort behandelt werden. 


\subsection{Palliative Netzwerke}

Regionale Netzwerke, die ein breites Spektrum an Institutionen, Versorgungsangeboten und wirksamer Koordination bieten, können den Zugang zu Palliativversorgung verbessern und die Qualität wie auch die Kontinuität der Betreuung steigern.

Relevante Expertenberichte zur Qualität der Palliativversorgung empfehlen die spezialisierte Palliativstation als Zentrum eines regionalen Palliativnetzwerks [2,3].

Es sollte mindestens eine spezialisierte Palliativstation in jedem regionalen Versorgungsgebiet zur Verfügung stehen.

Die spezialisierte Palliativstation sollte das Kernelement der spezialisierten Palliativversorgung sein. Die Angebote der spezialisierten Palliativversorgung in allen anderen Versorgungsstrukturen einschließlich allgemeiner Krankenhäuser und gemeindebasierter Primärversorgung sollten von der spezialisierten Palliativstation ausgehen oder formal mit ihr verbunden sein [3]. Diese Verbindung garantiert nicht nur die Unterstützung und kontinuierliche Fortbildung der Teams, sondern verbessert auch die Kontinuität der Patientenversorgung [2].

In seinen Empfehlungen stellt der Europarat fest, dass die Versorgungsqualität in einer bestimmten Region nicht nur von der Qualität der einzelnen Institutionen und Versorgungsangebote abhängt, sondern auch von der Koordination der spezialisierten Angebote und der Koordination zwischen den spezialisierten Angeboten und den Angeboten der Primärversorgung [2]. Wenn die Versorgungsangebote innerhalb eines zusammenhängenden regionalen Netzwerkes organisiert sind, verbessert dies den $\mathrm{Zu}$ gang zur Palliativversorgung und kommt sowohl der Qualität wie auch der Kontinuität der Betreuung zugute [9,11-13]. Damit ein solches Netzwerk gut funktioniert, müssen 2 Bedingungen erfüllt sein: eine wirksame Koordination und ein breites Spektrum an Institutionen und Dienstleistungen.

Die wirksame Koordination wird am besten durch einen CaseManager (Fallkoordinator, Schlüsselperson) erreicht, der den Informationsfluss und die Kontinuität der Versorgung über die verschiedenen Versorgungsstrukturen bewerkstelligen kann [5]. Eine wirksame Koordination der Versorgungsangebote wird es einem größeren Teil der Menschen ermöglichen, zu Hause zu sterben, falls sie dies wünschen. Um effektiv zu sein, müssen diese Koordinationsleistungen 24 Stunden am Tag und 7 Tage pro Woche verfügbar sein.

Die Koordination kann durch ein Team oder eine Person geleistet werden. Das Case-Management und die Koordination können von einer interdisziplinären Expertengruppe vorgenommen werden, die die verschiedenen Versorgungsangebote innerhalb des Netzwerks repräsentiert oder von einer Palliativstation oder einem stationären Hospiz.

Das Spektrum der Versorgungsangebote sollte alle verschiedenen Stufen der Versorgung, die von den Patienten benötigt werden, abdecken ( Abb.1) [10]. Zusätzlich werden Liaisondienste nachdrücklich empfohlen (Konsildienste im Krankenhaus sowie ambulante Palliativdienste).

Ein Palliativversorgungsnetzwerk hat mehrere Aufgaben. Komponenten eines gut funktionierenden Netzwerkes sind neben der Koordination der Betreuung:

- die gemeinsame Definition von Zielen und Qualitätsstandards

- einheitliche Aufnahme- und Entlassungskriterien auf allen Ebenen der Betreuung

- die Anwendung gemeinsamer Evaluationsmethoden

- die Einführung gemeinsamer therapeutischer Strategien auf der Grundlage der vorhandenen Evidenz
Über das Angebot der Betreuung hinaus sollte das Netzwerk die eigene Effizienz überprüfen, z. B. durch Audits, und sollte Ausbildungs- und Forschungsaktivitäten organisieren und supervidieren [14].

\subsection{Personal in der spezialisierten Palliativversorgung} Versorgungsangebote, die nicht in Palliativversorgung spezialisiert sind, können nach einem palliativen Versorgungsansatz vorgehen oder palliative Grundversorgung leisten, selbst wenn dafür nur eine einzelne Berufsgruppe oder gar eine einzelne Person (z. B. ein Hausarzt in einer Einzelpraxis) zur Verfügung steht, sofern sie von einem interdisziplinären Team unterstützt werden.

Am effektivsten wird Palliativversorgung von einem interdisziplinären Team von Behandlern durchgeführt, die sowohl über das Wissen als auch über die praktischen Erfahrungen in allen ihre Disziplin betreffenden Aspekten des Versorgungsprozesses verfügen [15]. In nicht spezialisierten Versorgungsstrukturen ist multiprofessionelle Teamarbeit allerdings eher die Ausnahme als die Regel. Es sollte daher sichergestellt sein, dass nicht spezialisierte Dienste kontinuierlich in einem angemessenen Austausch mit spezialisierten Anbietern stehen.

Für die spezialisierten palliativen Versorgungsangebote wird ein multiprofessionelles Team mit einem interdisziplinären Arbeitsstil benötigt.

Der Europarat [2] und das National Advisory Committee on Palliative Care [3] beschreiben die personellen Voraussetzungen für Angebote der spezialisierten Palliativversorgung. Demnach sollten die folgenden Fachkräfte - in Ergänzung zu Pflegefachkräften und ärztlichem Personal - in Vollzeit-, Teilzeittätigkeit oder regelmäßig zu festgelegten Zeiten zur Verfügung stehen:

- Sozialarbeiter

- Behandler mit Kompetenzen in psychosozialer Unterstützung

- eine angemessene Anzahl von Mitarbeitern für administrative und Sekretariats- sowie allgemeine Bürotätigkeiten

- Physiotherapeuten

- Trauerbegleiter

- Koordinatoren für seelsorgerische Betreuung

- Koordinatoren für Ehrenamtliche

- Seelsorger (Klinikseelsorge)

- Wundpflegespezialisten

- Spezialisten für Lymphödemtherapie

- Ergotherapeuten

- Logotherapeuten

- Ernährungsberater

> Pharmazeuten

- Therapeuten für komplementäre/alternative Heilverfahren

- Trainer/Unterrichtende

- Bibliothekare

Das palliative Kernteam sollte - als Minimalanforderung - aus Pflegefachkräften und Ärzten mit einer spezialisierten Ausbildung bestehen und, wenn möglich, durch Psychologen, Sozialarbeiter und Physiotherapeuten ergänzt werden. Behandler anderer Berufsgruppen können Mitglieder des Kernteams sein, häufiger wird es sich hierbei jedoch um Liaisondienste handeln.

Hierbei müssen Unterschiede zwischen verschiedenen europäischen Ländern, Regionen und Versorgungsstrukturen in Bezug auf die Mitglieder des Kernteams und des erweiterten Teams berücksichtigt werden. Zum Beispiel werden in manchen Ländern Psychologen als wichtige Mitglieder der Kernteams betrachtet, während in anderen Ländern diese Berufsgruppe für die klinische Arbeit mit Patienten und ihren Familien keine Bedeutung hat. 
Kinder benötigen spezialisierte Versorgungsangebote und diese müssen von pädiatrisch geschultem Personal umgesetzt werden. Dies gilt vor allem für pädiatrische Palliativpflegedienste. Häusliche Palliativangebote zur Unterstützung von Kindern und deren Familien sollten verfügbar sein [2].

\subsection{Ehrenamtliche Arbeit}

Einrichtungen der spezialisierten Palliativversorgung sollten Ehrenamtliche einschließen oder mit ehrenamtlichen Diensten zusammenarbeiten.

Ehrenamtliche Mitarbeiter leisten einen unverzichtbaren Beitrag zur Palliativversorgung. Die Hospizbewegung als Bürgerbewegung basiert auf ehrenamtlicher Arbeit. Der Europarat unterstreicht die Wichtigkeit der ehrenamtlichen Arbeit zur Ermöglichung hochqualitativer Palliativversorgung in der Gemeinde [2]. Damit ehrenamtliche Mitarbeiter die Rolle eines Partners im Palliativnetzwerk adäquat ausfüllen können, müssen einige Voraussetzungen gegeben sein:

- Ehrenamtliche Mitarbeiter müssen von einem entsprechenden Dachverband befähigt, supervidiert und anerkannt werden.

- Befähigung ist unabdingbar und verlangt eine sorgfältige Auswahl der ehrenamtlichen Mitarbeiter.

- Ehrenamtliche Mitarbeiter arbeiten innerhalb eines Teams unter der Verantwortung eines Koordinators.

- Der Koordinator ist die Verbindung zwischen den ehrenamtlichen Mitarbeitern und den Behandlern, zwischen dem Krankenhaus und dem Dachverband.

- Im interdisziplinären Team ersetzen die ehrenamtlichen Mitarbeiter kein Personal, sondern ergänzen die Arbeit des Teams.

Bezüglich der detaillierten Personalanforderungen für die unterschiedlichen Angebote der Palliativversorgung siehe Abschnitt 8 („Palliative Versorgungsangebote“).

\subsection{Palliative Pflegekarenz}

Die rechtlichen Voraussetzungen für eine Karenzzeit für pflegende Angehörige von Palliativpatienten sind nur in Österreich und Frankreich gegeben. Ähnliche Regelungen sollten auch in anderen europäischen Ländern eingeführt werden, um es Familienmitgliedern zu ermöglichen, ihre Angehörigen so lange wie erforderlich zu Hause zu betreuen.

Eine Beurlaubung vom Arbeitsplatz für Menschen, die für ihre schwerkranken und sterbenden Ehepartner, Eltern, Kinder oder andere nahe Verwandte sorgen, würde es vielen Patienten ermöglichen, bis zu ihrem Tod in ihrem Zuhause zu bleiben. Rechtliche Grundlagen für eine palliative Pflegekarenz existieren allerdings nur in 2 europäischen Ländern. Die Einführung von vergleichbaren Gesetzgebungen in anderen Ländern wäre ein großer Schritt in der Entwicklung der Palliativversorgung. Die rechtliche Anerkennung eines Erstattungsanspruchs für eine palliative Pflegekarenz würde zudem für Familienmitglieder und andere pflegende Angehörige die Belastung durch die Pflege mindern.

\section{Palliative Versorgungsangebote \\ $\nabla$}

In diesem Abschnitt werden Richtlinien zu den minimalen Ressourcen gegeben, die erforderlich sind, um dem Bedarf an Palliativversorgung in der Bevölkerung einer Region zu begegnen. Die verschiedenen Arten von Versorgungsangeboten werden anhand der folgenden Charakteristika vorgestellt:
- Definition und Zielsetzung der Angebote

- Bedarf an wohnortnaher Versorgung in der Region

- Ausstattung (Personal und räumliche Anforderungen)

\subsection{Palliativstation}

8.1.1 Definition und Zielsetzung

Palliativstationen erbringen die spezialisierte stationäre Palliativversorgung. Eine Palliativstation ist eine auf die Behandlung und Betreuung von Palliativpatienten spezialisierte Abteilung. Normalerweise handelt es sich um eine Abteilung innerhalb eines Krankenhauses oder eine diesem angegliederte Abteilung, aber sie kann auch als eigenständige Struktur vorkommen. In manchen Ländern sind Palliativstationen reguläre Stationen eines Krankenhauses, die Kriseninterventionen für Patienten mit komplexen Symptomen und Problemen anbieten; in anderen Ländern können Palliativstationen auch alleine arbeitende Institutionen sein, die Versorgung am Lebensende für Patienten leisten, die nicht länger zu Hause betreut werden können. Das Ziel der Palliativstationen ist es, krankheits- und therapiebedingte Beschwerden zu lindern und, falls möglich, den Zustand des Patienten zu stabilisieren sowie den Patienten und seine Angehörigen psychologisch und sozial so zu unterstützen, dass die Entlassung nach Hause oder die Verlegung in eine andere Versorgungsstruktur möglich wird.

Palliativstationen nehmen Patienten auf, deren medizinischer Zustand (körperlich, psychologisch, sozial und spirituell) spezialisierte multiprofessionelle Palliativversorgung erfordert [2]. Patienten können für wenige Tage oder für einige Wochen auf diese Stationen aufgenommen werden; medizinische, pflegerische, psychosoziale oder spirituelle Probleme können dafür vorrangig ausschlaggebend sein.

Palliativstationen können auch Betreuung in Tageshospizen, ambulante und häusliche Versorgung, Unterstützung bei der angemessenen Versorgung von Patienten in Alten- und Pflegeheimen und Trauerbegleitung anbieten, zusammen mit fachlicher Beratung und Fortbildung [16].

Die wesentlichen Dienstleistungen sollten 24 Stunden pro Tag an 7 Tagen pro Woche verfügbar sein [15]. Wenn möglich, sollten eine 24-Stunden-Telefonberatung für Behandler und eine 24Stunden-Telefonrufbereitschaft für dem Palliativteam bekannte ambulante Patienten und deren Angehörige eingerichtet sein.

Palliativstationen sollen mit verschiedenen Versorgungsangeboten im ambulanten und stationären Bereich zusammenarbeiten [2]. Sie arbeiten in einem Netzwerk zusammen mit Gesundheitszentren, Krankenhausstationen, Hausärzten, ambulanten Pflegediensten und Hospizen sowie mit anderen entsprechenden Versorgungsangeboten [6].

\subsubsection{Bedarf}

Es wird geschätzt, dass 50 Palliativbetten pro 1 Mio. Einwohner benötigt werden. Bei einer optimalen Größe von 8-12 Betten pro Einheit (Palliativstation) würde dies 5 Palliativstationen pro 1 Mio. Einwohner entsprechen. Neuere Schätzungen haben den Bedarf für Palliativ- und Hospizbetten auf $80-100$ benötigte Betten pro 1 Mio. Einwohner hochgesetzt.

Bis vor Kurzem wurde der Bedarf an Palliativbetten für Tumorpatienten auf 50 Betten pro 1 Mio. Einwohner geschätzt. Diese Berechnung berücksichtigt jedoch weder den Bedarf für nicht onkologische Patienten noch die steigende Prävalenz an chronischen Erkrankungen in der älter werdenden europäischen Bevölkerung [2]. Der aktuelle Bedarf beläuft sich möglicherweise auf die doppelte Anzahl an Betten. 
Die erforderliche Anzahl an Palliativbetten in einer bestimmten Region ist im Allgemeinen abhängig von der regionalen Demografie und der sozioökonomischen Entwicklung sowie der Verfügbarkeit anderer spezialisierter palliativer Versorgungsangebote, die z.B. Entlastungspflege und ambulante und häusliche Versorgung anbieten. Für Österreich wurden 60 Betten für 1 Mio. Einwohner empfohlen, den regionalen Bedingungen entsprechend verteilt [10]. In Irland empfiehlt das National Advisory Committee on Palliative Care, dass mindestens 80-100 Palliativbetten für 1 Mio. Einwohner zur Verfügung stehen sollten [3]. Der EAPC Atlas of Palliative Care in Europe untersucht die bestehende Anzahl Palliativbetten in verschiedenen europäischen Ländern [17]. Die geschätzte Anzahl von Palliativbetten in Westeuropa bewegt sich zwischen 10-20 Betten pro 1 Mio. Einwohner (Portugal, Türkei) und 50-75 Betten pro 1 Mio. Einwohner (Schweden, UK, Island, Luxemburg). Folglich muss die Anzahl der Betten sogar in vielen westeuropäischen Ländern erhöht werden - und die Situation in den meisten osteuropäischen Ländern ist noch deutlich schlechter.

\subsubsection{Anforderungen}

Palliativstationen benötigen ein hochqualifiziertes multiprofessionelles Team [2]. Von den Teammitgliedern der Palliativstationen wird erwartet, dass sie über eine spezialisierte Weiterbildung verfügen. Das Kernteam besteht aus Ärzten und Pflegefachkräften. Das erweiterte Team umfasst relevante angegliederte Professionen wie Psychologen, Physiotherapeuten, Sozialarbeiter oder Seelsorger.

Ein zentrales Merkmal der Palliativstation ist das multiprofessionelle Team mit spezialisierten Teammitgliedern aus verschiedenen Gesundheitsberufen, ergänzt durch ehrenamtliche Mitarbeiter [6].

Die Minimalanforderung an das Kernteam sind Ärzte und Pflegefachkräfte, dazu kommt regelhaft die Mitarbeit von Psychotherapeuten, Sozialarbeitern, Physiotherapeuten und Seelsorgern. Darüber hinaus sollten Behandler anderer Professionen bei Bedarf problemlos hinzugezogen werden können. Von allen Mitgliedern des Kernteams wird erwartet, dass sie über eine vom nationalen Berufsverband zertifizierte Weiterbildung in Palliativversorgung verfügen.

Zum ärztlichen Personal sollen Fachärzte mit entsprechendem Spezialwissen und Erfahrung in Palliativmedizin gehören. Von den Pflegefachpersonen wird erwartet, dass sie nach dem Examen eine professionelle Weiterbildung in Palliativversorgung abgeschlossen haben. Ehrenamtliche Mitarbeiter sollten einen Kurs für ehrenamtliche Hospizarbeit absolviert haben [10].

Palliativstationen benötigen ein fest zugeordnetes Kernteam von Pflegefachkräften und Ärzten. Auf Palliativstationen sollte das Verhältnis von Pflegefachkräften pro Bett mindestens $1: 1$, besser jedoch 1,2:1 betragen. Für Palliativstationen werden Ärzte mit einer spezialisierten Weiterbildung gebraucht, in einem Verhältnis von mindestens $\mathbf{0 , 1 5}$ Ärzten pro Bett.

In Versorgungsstrukturen, in denen Kinder betreut werden, sollte in jeder Schicht mindestens eine Pflegefachkraft mit spezieller pädiatrischer Qualifikation arbeiten.

Es gibt eine Vielzahl von Empfehlungen zur Personalausstattung hinsichtlich spezialisierter Palliativpflegekräfte für verschiedene Versorgungsstrukturen. So sollte nicht weniger als 1 spezialisierte Vollzeitpflegekraft pro Bett vorhanden sein [3]. Ebenso wird empfohlen, dass zumindest ein vollzeitbeschäftigter in Palliativmedizin spezialisierter Facharzt für 5-6 Betten zur Verfügung steht.
Palliativstationen benötigen ein erweitertes Team mit Vertretern anderer relevanter Berufsgruppen wie z. B. Psychologen, Sozialarbeitern, Seelsorgern oder Physiotherapeuten. Diese Behandler sollten ins Team integriert sein oder eng mit ihm zusammenarbeiten. Eine ständige Mitarbeit oder zumindest ein problemloses und geregeltes Hinzuziehen dieser Berufsgruppen bei Bedarf sollte gesichert sein.

Nationale Gutachten zu Normen für die Personalausstattung von Palliativstationen stimmen darin überein, dass folgende relevante Berufsgruppen in ein erweitertes Team der Palliativstation einbezogen sein müssen: Logotherapeuten, Psychologen, Krankenhausseelsorger, Ergotherapeuten, Ernährungsberater, Pharmazeuten, Physiotherapeuten, Wundpflegespezialisten, Spezialisten für Lymphödemtherapie, Sozialarbeiter und Spezialisten für Trauerbegleitung. Zusätzlich sollten ehrenamtliche Mitarbeiter mit einem hauptamtlichen Koordinator für ehrenamtliche Hospizarbeit zur Verfügung stehen.

Palliativstationen sollten eine heimische Atmosphäre mit ruhigen und privaten Bereichen bieten. Sie sollten separate Einheiten mit einer Kapazität von 8-15 Betten sein. Die Abteilung sollte mit 1oder 2-Bett-Patientenzimmern und Übernachtungsmöglichkeiten für Angehörige sowie Räumen für soziale Aktivitäten, z. B. Küche oder Wohnzimmer, ausgestattet sein.

Die Patientenbereiche sollten behindertengerecht ausgestattet sein. Jedes Patientenzimmer sollte ein eigenes Badezimmer haben. Auf der Abteilung sollte ein Raum zur Aufbahrung der Verstorbenen, zum Abschiednehmen und zum Meditieren sowie für multifunktionelle Nutzung zur Verfügung stehen. Der Zugang zu einer Kapelle oder einem Gebetsraum (Raum für Menschen mit unterschiedlichen Glaubensrichtungen) ist wünschenswert.

Das Versorgungssetting sollte Sicherheit und Bequemlichkeit sowie Möglichkeiten für Privatsphäre gewähren [15]. Wenn möglich, sollten die Versorgungsstrukturen eine heimische Atmosphäre und einen Zugang ins Freie bieten.

\subsection{Stationäres Hospiz \\ 8.2.1 Definition und Zielsetzung}

Ein stationäres Hospiz nimmt Patienten in ihrer letzten Lebensphase auf, wenn die Behandlung in einem Krankenhaus nicht mehr notwendig und die Betreuung zu Hause oder einem Pflegeheim nicht möglich ist.

Die zentralen Ziele eines stationären Hospizes sind die Linderung der Symptome und das Erreichen der bestmöglichen Lebensqualität bis zum Tod sowie Unterstützung in der Trauer [10].

In vielen Ländern haben stationäres Hospiz und Palliativstation eine sehr ähnliche Funktion, während in anderen Ländern klare Unterschiede zu erkennen sind.

In Deutschland werden z.B. Patienten für Kriseninterventionen auf eine Palliativstation aufgenommen, für eine Begleitung in der letzten Lebensphase hingegen in ein stationäres Hospiz. In manchen Ländern ist ein Hospiz im Gegensatz zu einer Palliativstation ein eigenständiges Versorgungsangebot, das den Schwerpunkt seiner Arbeit auf Versorgung am Lebensende (End-of-Life Care) legt.

\subsubsection{Anforderungen}

Ein stationäres Hospiz benötigt ein multiprofessionelles Team, das die Versorgung der Patienten und ihrer Angehörigen nach einem ganzheitlichen Ansatz leistet. Der Pflegepersonalschlüssel sollte mindestens 1, vorzugsweise 1,2 Pflegefachkräfte pro Bett betragen. Ein in Palliativmedizin qualifizierter Arzt sollte 24 Stunden am Tag erreichbar sein. Die psychosoziale und spirituelle Be- 
treuung durch entsprechend ausgebildete Mitarbeiter sollte regelhaft gewährleistet sein, ebenso sollten ehrenamtliche Mitarbeiter einbezogen werden.

Das Kernteam des stationären Hospizes besteht aus Pflegefachpersonen und muss stets rasch auf einen qualifizierten Arzt zugreifen können. Das erweiterte Team umfasst Sozialarbeiter, Psychologen, Seelsorger, Physiotherapeuten, Ernährungsberater, Logotherapeuten und Ergotherapeuten sowie ehrenamtliche Mitarbeiter [10].

Ein stationäres Hospiz erfordert eine heimische Atmosphäre mit behindertengerechter Gestaltung, 1- oder 2-Bett-Zimmern und einer Kapazität von mindestens 8 Betten. Das Hospiz sollte mit Räumlichkeiten für soziale und therapeutische Aktivitäten ausgestattet sein.

Ein stationäres Hospiz sollte eine eigenständige organisatorische Einheit mit einem eigenen Team und mindestens 8 Betten sein [10]. Die Patientenzimmer sollten mit einem Bad ausgestattet sein. Übernachtungsmöglichkeiten für Angehörige sollten angeboten werden.

\subsection{Palliativer Konsildienst ${ }^{5}$}

\subsubsection{Definition und Zielsetzung}

Der palliative Konsildienst leistet spezialisierte palliative Fachberatung und Unterstützung für anderes Klinikpersonal, Patienten und deren Familien sowie andere Behandler in der Versorgungsstruktur des Krankenhauses. Er bietet formelle und informelle Ausbildung an und vernetzt sich mit anderen Diensten innerhalb und außerhalb des Krankenhauses.

Palliative Konsildienste sind in einigen Ländern auch bekannt als Hospital Supportive Care Teams oder Hospital Mobile Teams [17]. Palliativkonsilteams bieten in erster Linie Unterstützung für Behandler in anderen Krankenhausabteilungen und Polikliniken, die nicht in Palliativversorgung spezialisiert sind [10].

Ein zentrales Ziel des Palliativkonsilteams ist die Linderung der zahlreichen Symptome der Palliativpatienten in verschiedenen Abteilungen des Krankenhauses durch ein Mentoring des diensthabenden Personals und durch Unterstützung der Patienten und ihrer Angehörigen. Des Weiteren soll die Expertise in Palliativmedizin und -versorgung in diesem Umfeld zur Verfügung gestellt werden.

Unterstützung und Fortbildung wird in den Bereichen Schmerztherapie, Symptomkontrolle, ganzheitliche Versorgung und psychosoziale Unterstützung angeboten. Dies beinhaltet die konsiliarische Mitbetreuung von Patienten auf einer Vielzahl von verschiedenen Abteilungen und die Beratung anderer Behandler. Die Entscheidung über und die Umsetzung von Therapien und Interventionen bleibt dabei jedoch in der Verantwortung des betreuenden medizinischen Personals. Das Palliativkonsilteam interveniert auf Anfrage des medizinischen oder pflegerischen Personals, des stationär aufgenommenen Patienten und seiner Angehörigen [2]. Vom Team wird erwartet, dass es mit anderen Spezialisten eng zusammenarbeitet.

Das Ziel eines Palliativkonsilteams ist die Verbesserung der Betreuung, um die Entlassung von der Akutstation zu fördern und den Übergang von stationärer zu ambulanter Betreuung zu vereinfachen.

Maßnahmen seitens des Teams können ein gut begleiteter Wechsel des Patienten auf die Palliativstation oder die Koordination

\footnotetext{
5 In Österreich und der Schweiz wird hauptsächlich der Begriff Palliativkonsiliardienst verwendet $[10,21]$.
}

zwischen stationärer und ambulanter Versorgung sein. Die enge Zusammenarbeit mit anderen Versorgungsangeboten und Beteiligung an einem tragfähigen palliativen Netzwerk sind wichtige Funktionen des Palliativkonsilteams. Mögliche zusätzliche Aufgaben in diesem Zusammenhang sind das Angebot einer Hotline zur Beratung von ärztlichem und pflegerischem Personal in schwierigen palliativen Fragestellungen und regionale Fortbildungs- und Trainingsangebote zu ausgewählten Fragestellungen der Palliativversorgung [6].

\subsubsection{Bedarf}

Ein palliativer Konsildienst sollte an jede Palliativstation angegliedert sein und sollte bei Bedarf jedem Krankenhaus zur Verfügung stehen. Für ein Krankenhaus mit 250 Betten sollte mindestens ein Team vorhanden sein.

Es wäre wünschenswert, wenn an jede Palliativstation ein Palliativkonsilteam angegliedert wäre. Im Bedarfsfall sollte ein solches Team für jedes Krankenhaus zur Verfügung stehen [10]. Allerdings ist dieses Unterstützungs- und Betreuungsmodell in der Mehrzahl der europäischen Länder noch sehr unterrepräsentiert [17]. Länder mit einem hohen Anteil an Palliativkonsilteams sind Belgien, Frankreich, Irland und UK. In den meisten anderen europäischen Ländern ist ein beträchtlicher Mangel an diesen Teams zu verzeichnen.

\subsubsection{Anforderungen}

Ein Palliativkonsilteam besteht aus einem multiprofessionellen Team mit mindestens einem Arzt und einer Pflegefachkraft mit spezialisierter Weiterbildung in Palliativversorgung.

Dem Kernteam sollten Ärzte und Pflegefachkräfte fest angehören $[10,16]$. Vom ärztlichen und pflegerischen Personal wird eine spezialisierte, durch die nationalen Fachgesellschaften akkreditierte Weiterbildung erwartet. Das Team sollte rasch andere Berufsgruppen hinzuziehen können, die in Liaison mit ihm arbeiten: Spezialisten in Trauerbegleitung, Krankenhausseelsorger, Ernährungsberater, Therapeuten, Onkologen, Pharmazeuten, Physiotherapeuten, Psychiater, Psychologen, Sozialarbeiter und Logotherapeuten. Das klinische Personal sollte durch administratives Personal unterstützt werden. Unterstützung der Mitarbeiter, z.B. durch Supervision, sollte dem Team zur Verfügung stehen.

Ein Palliativkonsilteam sollte einen Raum für Teamkonferenzen sowie administrative Unterstützung zur Verfügung haben.

\subsection{Ambulanter Palliativdienst ${ }^{6}$}

\subsubsection{Definition und Zielsetzung}

Das ambulante Palliativteam leistet spezialisierte Palliativversorgung für Patienten, die einer Versorgung zu Hause bedürfen und unterstützt deren Familien und Betreuer. Ebenso bietet es spezialisierte Beratung für Hausärzte, andere niedergelassene Fachärzte und Pflegefachpersonen, die die Patienten zu Hause betreuen. Das ambulante Palliativteam ist ein multiprofessionelles Team, das in erster Linie Menschen zu Hause oder im Pflegeheim (z. B. Verwandte, ärztliches Personal, Pflegefachpersonal, Physiotherapeuten) unterstützt. Es bietet Unterstützung nach einem abgestuften Versorgungsansatz. Meistens hat das ambulante Palliativteam eine Konsiliar- und Mentorfunktion und bietet Expertise in Schmerztherapie, Symptomkontrolle, Palliativversorgung und psychosozialer Unterstützung. Das ambulante Team kann die Be-

\footnotetext{
${ }^{6}$ In der Schweiz und in Österreich ist der Begriff „Mobiler Palliativdienst“ gebräuchlich und die Teams bestehen aus auf Palliativversorgung spezialisierten Fachleuten mit oder ohne ehrenamtliche Mitarbeiter [10,21].
} 
ratung und Unterstützung auch dem Patienten direkt anbieten. Weniger häufig ist es selbst direkt in die Pflege involviert - dann in Kooperation mit dem Hausarzt und anderen Primärversorgern. In einzelnen Fällen mit komplexen Symptomen und Problemen kann das ambulante Palliativteam die Behandlung vom Hausarzt und den Primärversorgern übernehmen und eine ganzheitliche Palliativversorgung leisten. Die Vorgehensweise ist auch abhängig von der lokalen Versorgungssituation und dem Grad, in dem primäre Behandler involviert sind. Das ambulante Palliativteam assistiert ebenfalls beim Transfer zwischen Krankenhaus und häuslicher Versorgung [10].

\subsubsection{Bedarf}

Es sollte ein ambulantes Palliativteam für 100000 Einwohner geben. Das Team sollte 24 Stunden pro Tag verfügbar sein [10].

Ambulante Palliativteams müssen an 7 Tagen pro Woche und 24 Stunden pro Tag verfügbar sein [6].

Gemäß dem EAPC Atlas of Palliative Care in Europe haben UK, Polen und Schweden einen hohen Anteil an ambulanten Palliativdiensten mit einem Verhältnis von ungefähr einem Dienst für 160000-180000 Einwohner [17]. Italien und Spanien verfügen ebenfalls über eine beachtliche Anzahl an ambulanten Diensten mit einem Verhältnis von ungefähr 1 Dienst auf 310000-380000 Einwohner. Die Anzahl der ambulanten Versorgungsangebote in den anderen europäischen Ländern ist allerdings deutlich kleiner. In einigen Regionen gibt es praktisch gar keine ambulanten Palliativdienste (z.B. gibt es in Deutschland insgesamt 30 Dienste für eine Bevölkerung von mehr als 80 Mio. Einwohnern).

In ländlichen Gegenden müssen ambulante Dienste größere Einzugsgebiete abdecken, um kosteneffektiv zu arbeiten [18]. Deshalb müssen regionale Netzwerke entwickelt werden, um ambulante Palliativversorgung in den ländlichen Gebieten mit geringer Infrastruktur anzubieten.

\subsubsection{Anforderungen}

Das Kernteam eines ambulanten Palliativteams besteht aus 4-5 vollzeitbeschäftigten Mitarbeitern und umfasst Ärzte und Pflegefachkräfte mit spezialisierter Weiterbildung, einen Sozialarbeiter und administratives Personal.

Das Kernteam eines mobilen ambulanten Palliativteams sollte aus Behandlern mit einer durch die nationalen Berufsverbände anerkannten spezialisierten Weiterbildung in Palliativversorgung bestehen [10].

In manchen ambulanten Palliativteams wird die tägliche Betreuung der Patienten und ihrer pflegenden Angehörigen von Personal ohne spezialisierte Weiterbildung geleistet, wobei diesem in Palliativversorgung spezialisierte Ärzte und Pflegefachkräfte zur fachlichen Anleitung und Supervision zur Verfügung stehen [7]. Zusätzlich sollten andere Berufsgruppen wie Physiotherapeuten, Psychologen, Ergotherapeuten, Logotherapeuten, Ernährungsberater und Seelsorger problemlos hinzugezogen werden können und ehrenamtliche Mitarbeiter vorhanden sein.

Das ambulante Palliativteam arbeitet eng mit anderen Berufsgruppen zusammen, sodass das gesamte Spektrum einer multiprofessionellen Teamarbeit im häuslichen Umfeld angeboten werden kann.

In manchen Ländern gibt es keine multiprofessionellen Teams, stattdessen wird die häusliche Palliativversorgung übernommen von spezialisierten Palliativpflegediensten und spezialisierten Ärzte, die in (Hausarzt-)Praxen oder in ambulanten Unterstützungsdiensten arbeiten.
Palliativversorgung im häuslichen Umfeld erfordert eine enge Zusammenarbeit mit anderen professionellen Dienstleistern wie spezialisierten Pflegediensten und niedergelassenen Ärzten mit spezialisierter Ausbildung einschließlich (aber nicht begrenzt auf) regelmäßige Besprechungen am Patientenbett (und andere Aufgaben).

Das ambulante Palliativteam benötigt einen Arbeitsraum, der den Pflegefachkräften, Ärzten und Sozialarbeitern zur Verfügung steht, sowie ein Besprechungszimmer und einen Lagerraum für medizinisches Material.

\section{5 „Krankenhaus zu Hause“ (Hospital at home)}

Das „Krankenhaus zu Hause“ bietet eine intensive, krankenhausartige Betreuung für den Patienten zu Hause.

In manchen europäischen Ländern, z.B. Frankreich oder Finnland, bietet das „Krankenhaus zu Hause“ eine intensive medizinische und pflegerische Versorgung, die es dem Patienten, der ansonsten ins Krankenhaus aufgenommen werden müsste, erlaubt, zu Hause zu bleiben. Dies erfordert eine Art der Betreuung, die der Versorgung im Krankenhaus viel ähnlicher ist als der üblichen häuslichen Versorgung. Es finden sich verschiedene organisatorische Modelle, die von einer Erweiterung der bestehenden Ressourcen im häuslichen Umfeld bis zur Zuteilung eines spezialisierten Teams reichen, das alle Anforderungen abdecken kann [2].

\subsection{Ambulanter Hospizdienst ${ }^{7}$ (ehrenamtliches Hospizteam) \\ 8.6.1 Definition und Zielsetzung}

Der ambulante Hospizdienst besteht aus ehrenamtlichen Mitarbeitern und bietet Unterstützung und Beistand für Palliativpatienten und deren Angehörige in Zeiten der Erkrankung, der Schmerzen, des Abschieds und der Trauer. Der ambulante Hospizdienst ist Teil eines umfassenden Unterstützungsnetzwerks und arbeitet eng mit professionellen Angeboten der Palliativversorgung zusammen.

Der ambulante Hospizdienst ist für die psychosoziale und emotionale Unterstützung der Patienten, Angehörigen und der professionellen Behandler wesentlich und fördert die Aufrechterhaltung und Verbesserung der Lebensqualität des Patienten und seiner Angehörigen. Die Unterstützung bleibt über den Tod des Patienten hinaus bestehen und geht in der Phase der Trauerbegleitung weiter [10]. Ambulante Hospizdienste leisten nicht nur einen unverzichtbaren Anteil an der Palliativversorgung für Patienten und deren Angehörige, sondern treten auch als Fürsprecher der Palliativversorgung in der Öffentlichkeit auf. In manchen Ländern beteiligen sich ehrenamtliche Mitarbeiter an der Akquisition von finanziellen Mitteln, an Repräsentations- und Verwaltungsaufgaben sowie an der Organisation und Leitung von Hospizen.

\subsubsection{Bedarf}

Es sollte ein ambulanter Hospizdienst für $\mathbf{4 0 0 0 0}$ Einwohner verfügbar sein [10].

Allerdings war der Konsens bezüglich dieser Aussage im DelphiProzess (s. Teil 1 dieses Artikels in Z Palliativmed 2011; 12: 216227) nicht so groß wie bei anderen Aussagen. Der Bedarf an ambulanten Hospizdiensten kann je nach der Rolle der ehrenamtlichen Mitarbeiter in den nationalen und lokalen Versorgungs-

\footnotetext{
${ }^{7}$ Bezeichnung in der Schweiz und Österreich: Freiwillige oder Hospizgruppe bzw. Hospizteam [10,21].
} 
strukturen unterschiedlich ausgeprägt sein. In manchen Regionen kann ein ambulantes Hospizteam für bis zu 80000 Einwohner ausreichend sein.

\subsubsection{Anforderungen}

Der ambulante Hospizdienst besteht aus speziell ausgebildeten ehrenamtlichen Hospizarbeitern und mindestens einem hauptamtlichen Koordinator.

Ein ambulanter Hospizdienst besteht aus mindestens 10-12 ehrenamtlichen Hospizarbeitern und einem festen hauptamtlichen Koordinator. Der Koordinator sollte eine Ausbildung im Bereich der Sozial- oder Gesundheitsversorgung und eine zusätzliche spezielle Schulung in Palliativversorgung haben. Die ehrenamtlichen Mitarbeiter sollten einen akkreditierten Lehrgang absolviert haben und an regelmäßigen Supervisionen und Selbstreflexionen sowie an einer kontinuierlichen Fortbildung teilnehmen [10]. Die erforderliche Qualifikation für ehrenamtliche Mitarbeiter und deren Koordinator ist abhängig von deren Arbeitsfeldern in der Palliativversorgung. Ehrenamtliche Mitarbeiter in administrativen oder anderen Funktionen bedürfen einer anderen Art von Schulung.

\subsection{Tageshospiz}

\subsubsection{Definition und Zielsetzung}

Tageshospize oder Tageszentren sind Orte in Krankenhäusern, stationären Hospizen, Palliativstationen oder Gemeinden, die speziell dafür geschaffen wurden, kreative und therapeutische Aktivitäten bei Palliativpatienten zu fördern.

Patienten verbringen normalerweise einen Teil des Tages im Tageszentrum, entweder jeden Tag oder einmal pro Woche. Tageshospize legen den Schwerpunkt ihrer Betreuung auf das kreative Erleben und die soziale Betreuung, wobei sie den Patienten die Möglichkeit bieten, während des Tages an verschiedenen Aktivitäten außerhalb des familiären Umfeldes teilzunehmen. Formale ärztliche Betreuung ist üblicherweise nicht Teil der täglichen Routine, jedoch können Patienten in manchen Tagesbetreuungszentren einige Behandlungen, wie z.B. Bluttransfusionen oder eine Chemotherapie, erhalten, während sie im Zentrum sind.

Das zentrale Anliegen ist eine soziale und therapeutische Betreuung, um die soziale Isolation zu verhindern sowie die Belastung der Angehörigen und der Betreuungspersonen zu verringern.

\subsubsection{Bedarf}

Bis heute sind Tageszentren ein charakteristisches Kennzeichen der Hospiz- und Palliativentwicklung in Großbritannien mit einer angegebenen Zahl von mehr als 200 Zentren. In anderen europäischen Ländern sind sie jedoch nur sehr spärlich vertreten [19]. Demzufolge fehlt eine klare Schätzung des Bedarfs an Tageszentren in europäischen Ländern. Eine mittlere Voraussage des Bedarfs an palliativen Versorgungsangeboten empfiehlt jedoch ein Einzugsgebiet von 150000 Einwohnern für ein Tageszentrum. Aufgrund der mangelnden Evidenz werden Pilotprojekte stark angeraten [10].

Es muss angemerkt werden, dass in vielen Ländern Tageshospize nicht als wesentliches Versorgungsangebot betrachtet werden und stattdessen andere Modelle palliativer Versorgungsleistungen angeboten werden.

Es sollte ein Tageshospiz für 150000 Einwohner zur Verfügung stehen [10]. Nationale Pilotprojekte sollten aufgebaut und evaluiert werden, um den Bedarf für diese Art von Versorgungsangebot zu untersuchen.

\subsubsection{Anforderungen}

Ein Tageszentrum verfügt über ein multiprofessionelles Team und wird von ehrenamtlichen Mitarbeitern unterstützt. Es wird empfohlen, dass 2 Pflegefachkräfte während der Öffnungszeiten präsent sind, wobei mindestens eine spezialisierte Palliativpflegefachkraft für 7 Tagesbesucher zur Verfügung steht. Ein qualifizierter Arzt sollte im Bedarfsfall direkt erreichbar sein. Ein schnelles Hinzuziehen anderer Professionen wie Physiotherapeut, Sozialarbeiter oder Seelsorger sollte gewährleistet sein [3,10].

Ein Tageshospiz sollte Patientenräume, einen Therapieraum, Personalräume, ein Bad, eine Küche und einen Ruheraum haben. Alle Räume sollten behindertengerecht gestaltet sein.

Ein Tageszentrum sollte eine heimische Atmosphäre bieten. Die Patientenräume sollten bequem ausgestattet und für multifunktionellen Gebrauch geeignet sein. Das Zentrum ist eine eigenständige organisatorische Einheit mit mindestens 6 Plätzen und kann einem stationären Hospiz oder einer Palliativstation angegliedert sein.

\subsection{Palliativambulanz (Palliative Outpatient Clinic)}

Palliativambulanzen bieten Sprechstunden und Beratung für Patienten, die zu Hause leben und denen es möglich ist, die Klinik aufzusuchen.

Palliativambulanzen sind ein wichtiger Bestandteil der gemeindenahen Palliativversorgungsprogramme. Normalerweise sind sie an spezialisierte Palliativstationen oder stationäre Hospize angegliedert. Patienten mit fortschreitenden Erkrankungen und eingeschränktem Allgemeinzustand ist es oft nicht länger möglich, in die Ambulanz zu kommen. Deshalb sollten Palliativambulanzen in die regionalen Netzwerke integriert sein, um sich mit stationären Diensten, ambulanten palliativen Teams oder Primärversorgerteams abzustimmen.

\section{Diskussion und Ausblick}

Nach einer Pionierphase von ungefähr 4 Jahrzehnten ist die Palliativversorgung noch immer eine ziemlich junge Disziplin, und Konsensusprozesse sind eine neue Entwicklung. Ferris et al. halten in ihrer Publikation über das US-basierte Modell zur Anleitung der Versorgung von Patienten und ihren Familien fest, dass grundlegender Fortschritt sich dort entwickeln konnte, wo es Vorkämpfer gab [15]. Die Versorgungsansätze gründeten üblicherweise auf individuellen Auffassungen. Es ließ sich eine beachtliche Variabilität sowohl der individuellen Versorgungsprogramme als auch der uneinheitlichen Zugangsmöglichkeiten zur Versorgung beobachten.

In ihrer jüngsten Publikation der Resultate der EAPC Task Force zur Entwicklung der Palliativversorgung in Europa zeigten Carlos Centeno u. Kollegen die folgenden Probleme bei der Evaluation von Angeboten der Palliativversorgung auf [19]:

- verschiedene Arten von Versorgungsangeboten in verschiedenen Ländern

- Mangel an einheitlichen Standards und allgemein akzeptierten Definitionen aller verfügbaren Arten von Versorgungsangeboten

- verschiedene Interpretationen dessen, was als „spezialisierte palliative Versorgungsressource" betrachtet wird

Mit Bezug auf diese Publikation stellte von Gunten fest, dass diese Inkonsistenz den Dialog, den Vergleich und den Fortschritt behindert [20]. Er plädierte stark für einen europäischen Konsens 
und wies auf Kanada und die USA hin, die mit gutem Beispiel vorangegangen sind.

Der Bedarf an fachlichem Konsens darüber, was hochqualifizierte Palliativversorgung ausmacht, ist eine Voraussetzung für eine effektive Versorgungsleistung innerhalb des gesamten Betreuungsspektrums.

Diese von der EAPC empfohlenen Richtlinien und Standards zu verbreiten und gegenüber Entscheidungsträgern und Interessensvertretern zu befürworten, wird das öffentliche Interesse an der Hospiz- und Palliativversorgung in Europa stärken [4].

Die World Health Organization (WHO) empfiehlt Politikern, sicherzustellen, dass Palliativversorgung ein integraler Bestandteil jeder Gesundheitsversorgung wird und nicht als „zusätzliches Extra“ gesehen wird [5]. Dies steht in Übereinstimmung mit den Empfehlungen des Europarates an seine Mitgliedstaaten, dass Palliativversorgung ein unentbehrlicher und integraler Teil des Gesundheitssystems ist. Deshalb sollten nationale Gesundheitsstrategien Maßnahmen zur Entwicklung und tragfähigen Integration der Palliativversorgung beinhalten [2]. Gesundheitsorganisationen müssen für die Qualitätsverbesserung belohnt werden. Partnerschaften zwischen geriatrischen Teams, Pflegeheimen und Behandlern der Palliativversorgung sollten aufgebaut werden.

Die EAPC unterstützt die folgenden an Politiker und Entscheidungsträger gerichteten Empfehlungen der WHO [5]:

- Anerkennung der gesundheitspolitischen Bedeutung der alternden Bevölkerung

- Durchführung von Qualitätsaudits palliativer Versorgungsangebote

- Förderung der Entwicklung von Kerndatenpaketen

- Förderung von Audits und Qualitätsverbesserungsmethoden/ Honorieren der Einbeziehung von Organisationen im Gesundheitswesen

- Sicherstellung einer adäquaten Finanzierung, Honorierung und Unterstützung multiprofessioneller Versorgungsangebote

- Sicherstellung eines ausreichenden Zeitdeputats für palliative Lerninhalte in der Weiterbildung von Berufen im Gesundheitswesen und Unterstützung der Behandler, sich regelmäßig auf den neuesten Stand zu bringen

- gezieltes Vorgehen gegen die Stereotypen, die dazu führen, dass älteren Menschen im Bedarfsfall keine Palliativversorgung angeboten wird

Der Schwerpunkt dieses Weißbuches liegt auf den Richtlinien und Standards für Hospiz- und Palliativversorgung in Europa gemäß den Empfehlungen der EAPC. Der Inhalt wurde auf die Aspekte beschränkt, die direkt mit der Verteilung von Versorgungsangeboten auf Gemeindeebene zusammenhängen sowie mit der technischen, räumlichen und personellen Ausstattung dieser Versorgungsangebote.

Selbstverständlich spielen zusätzlich zur Struktur der Versorgung andere Dimensionen für die Bereitstellung hochqualitativer Hospiz- und Palliativversorgung eine wichtige Rolle - beispielsweise Behandlungsmethoden, Qualitäts- und Outcome-Assessment, Forschung, Ausbildung, Finanzierung, Politik und Organisation sowie rechtliche Regelungen. Diese Bereiche im Detail abzudecken, übersteigt die Möglichkeiten dieses Weißbuchs. Relevante Themen werden in einer Reihe zukünftiger Publikationen eingehender beleuchtet.

\section{Danksagung}

Dieses Forschungsprojekt wurde durch eine nicht zweckgebundene Zuwendung seitens der Klinik für Palliativmedizin der RWTH Aachen (Deutschland) unterstützt. Das Manuskript wurde von Saskia Jünger vorbereitet. Wir möchten den Experten danken, die Zeit und Mühe in die kritische Durchsicht des Manuskripts investiert haben: Franco De Conno, Carl-Johan Fürst, Geoffrey Hanks, Irene Higginson, Stein Kaasa, Phil Larkin und Friedemann Nauck. Wir möchten uns ebenfalls bei den Vorständen der nationalen Fachgesellschaften für ihre engagierte Beteiligung am Delphi-Prozess bedanken. Ohne ihr Engagement und ihre Unterstützung wäre es nicht möglich gewesen, diese Empfehlungen in so kurzer Zeit zu entwickeln.

\section{Literatur}

1 Kaas-Bartelmes BL, Hughes R, Rutherford MK. Advance care planning: preferences for care at the end of life. Rockville: US Department of Health and Human Service, Agency for Healthcare Research and Quality; 2003

2 Council of Europe. Recommendation Rec (2003) 24 of the Committee of Ministers to member states on the organisation of palliative care, 2003; im Internet: www.coe.int/t/dg3/health/Source/Rec(2003)24_en. pdf; Stand: 30.09.2011

3 Mid-Western Health Board. Report on the National Advisory Committee on Palliative Care. Department of Health and Children; 2001; im Internet: http://hdl.handle.net/10147/43031; Stand: 30.09.2011

4 National Consensus Project for Quality Palliative Care. Clinical Practice Guidelines for Quality Palliative Care, 2004

5 Davies E, Higginson IJ, eds. Better Palliative Care for Older People. Copenhagen: World Health Organization Regional Office for Europe; 2004

6 Jaspers B, Schindler T. Stand der Palliativmedizin und Hospizarbeit in Deutschland und im Vergleich zu ausgewählten Staaten (Belgien, Frankreich, Großbritannien, Niederlande, Norwegen, Österreich, Polen, Schweden, Schweiz, Spanien). Berlin: Enquete-Kommission des Bundestages „Ethik und Recht der modernen Medizin“; 2005

7 National Institute for Health and Clinical Excellence. Improving supportive and palliative care for adults with cancer. The manual, 2004; im Internet: www.nice.org.uk/nicemedia/pdf/csgspmanual.pdf; Stand: 30.09.2011

8 Tomlinson S. National Action Planning Workshop on End-of-life Care. Winnipeg: Health Canada Secretariat on Palliative and End-of-life Care; 2002

9 Ellershaw J. Care of the dying: what a difference an LCP makes! Palliat Med 2007; 21: 365-368

10 Nemeth C, Rottenhofer I. Abgestufte Hospiz- und Palliativversorgung in Österreich. Wien: Österreichisches Bundesinstitut für Gesundheitswesen; 2004

11 Elsey B, McIntyre J. Assessing a support and learning network for palliative care workers in a country area of South Australia. Aust J Rural Health 1996; 4: 159-164

12 Mitchell G, Price J. Developing palliative care services in regional areas. The Ipswich Palliative Care Network model. Aust Famy Physician 2001; 30: 59-62

13 Schroder C, Seely JF. Pall-Connect: a support network for community physicians. J Palliat Care 1998; 14: 98-101

14 Gómez-Batiste X, Porta-Sales J, Pascual A et al. Palliative Care Advisory Committee of the Standing Advisory Committee for Socio-Health Affairs, Department of Health, Government of Catalonia. Catalonia WHO palliative care demonstration project at 15 years (2005). J Pain Symptom Manage 2007; 33: 584-590

15 Ferris FD, Balfour HM, Bowen Ket al. A model to guide patient and family care: based on nationally accepted principles and norms of practice. J Pain Symptom Manage 2002; 24: 106-123

16 Clinical Standards Board for Scotland. Clinical standards: specialist palliative care. Edinburgh: 2002

17 Centeno C, Clark D, Lynch T et al. EAPC Atlas of Palliative Care in Europe. Houston: IAHPC Press; 2007

18 Radbruch $L$, Voltz R. Was ist speziell an der spezialisierten Palliativversorgung? Der Gemeinsame Bundesausschuss legt Beschlüsse zur spezialisierten ambulanten Palliativversorgung vor. Der Schmerz 2008; 22: 7-8 
19 Centeno C, Clark D, Lynch T et al. Facts and indicators on palliative care development in 52 countries of the WHO European region: results of an EAPC task force. Palliat Med 2007; 21: 463-471

20 Gunten CF von. Humpty-Dumpty Syndrome. Palliat Med 2007; 21: 461-462
21 Eychmüller S. Versorgungsstrukturen Palliative Care Schweiz. palliative ch/Schweizerische Gesellschaft für Palliative Medizin, Pflege und Begleitung; im Internet: http://www.palliative.ch/fileadmin/user_up load/palliative/fachwelt/E_Standards/versorgungsstrukturen_de.pdf; Stand: 30.09.2011 